\title{
SDR IMPLEMENTATION OF A TESTBED FOR SYNCHRONIZATION OF COHERENT DISTRIBUTED REMOTE SENSING SYSTEMS
}

\author{
${ }^{1}$ J. C. Merlano-Duncan, ${ }^{1}$ J. Querol, ${ }^{1}$ L. Martinez-Marrero, ${ }^{1}$ J. Krivochiza, ${ }^{2}$ A. Camps, ${ }^{1}$ S. Chatzinotas, \\ ${ }^{1}$ B. Ottersten
}

\author{
${ }^{1}$ Interdisciplinary Centre for Security Reliability and Trust (SnT), University of Luxembourg \\ ${ }^{2}$ Unidad María de Maeztu CommSensLab - Dept. of Signal Theory and Communications, Universitat \\ Politécnica de Catalunya-BarcelonaTech and IEEC/CTE-UPC \\ Email: \{juan.duncan, jorge.querol, liz.martinez-marrero, jevgenij.krivochiza, symeon.chatzinotas, \\ bjorn.ottersten\}@uni.lu; \\ camps@tsc.upc.edu
}

\begin{abstract}
Remote Sensing from distributed platforms has become attractive for the community in the last years. Phase, frequency, and time synchronization are a crucial requirement for many such applications as multi-static remote sensing and also for distributed beamforming for communications. The literature on the field is extensive, and in some cases, the requirements an complexity of the proposed synchronization solution may surpass the ones set by the application itself. Moreover, the synchronization solution becomes even more challenging when the nodes are flying or hovering on aerial or space platforms. In this work, we discuss the synchronization considerations for the implementation of distributed remote sensing applications. The general framework considered is based on a distributed collection of autonomous nodes that synchronize their clocks with a common reference using inter-satellite links. For this purpose, we implement a synchronization link between two nodes operating in a full-duplex fashion. The experimental testbed uses commercially available SDR platforms to emulate two satellites, two targets, and the communication channel. The proposal is evaluated considering phase and frequency errors for different system parameters.
\end{abstract}

Index Terms - Synchronization, multi-static remote sensing systems, distributed beamforming, SDR implementation.

\section{INTRODUCTION}

During the last years, the earth observation community has increased its interest in distributed missions deployed in more than one platform, in flying formations using smaller and low-cost vehicles. Some examples of distributed remote sensing applications are the bistatic and multi-static SAR [1], in which the Tandem- $X$ was the first companion SAR concept mission[2]. Another recent example is the European Space Agency Harmony Mission (within the Earth Explorer 10 program), in which two identical receive-only spacecraft follow in a formation to the Sentinel-1D and use it as a radar illuminator [3]. On the other hand, multistatic configurations are the only real alternatives to achieve the radar power budget in missions from MEO to GEO orbits [4][5][6].

Similarly, the distributed and formation flying configurations have been considered for applications in radiometry. The spatial resolution of a single platform radiometer can be improved only by increasing its aperture size. Therefore, the use of formation flying configurations provides the potential to increase spatial resolution significantly [7][8][9]. The use of this technique is foreseen to be applied in 3D synthetic apertures, in contrast to 2D coplanar arrays [10], providing the system with more flexibility, and giving the possibility to reduce the mutual coupling between the antennas.

The synchronization in these systems becomes extremely challenging when the distance between the distributed nodes is significant in terms of the signal wavelengths and, in particular, when this electrical distance is varying in time due to fast changes in the conditions of the transmission media and in the RF components of the array elements [11]. In particular, the RF components add phase and timing drifts that can not be easily parametrized or characterized, and they represent an impediment to the implementation of distributed coherent applications such as beamforming [11].

On the other hand, in the practical implementation of the round trip synchronization between two nodes, if the transmitted by each of the two nodes use different carrier frequencies, then the loop will not achieve a perfect synchronization since the drifts are different and cannot be compensated. Therefore, a round trip loop using the same carrier, known as In-Band Full-Duplex (IBFD), would solve both of the above problems. First, it would better control the drifts by assuming reciprocity in the paths of the back and forth signals. And second, it would help each node to receive and calibrate its self signal, eliminating the unknown drifts produced by its RF channel. The use of IBFD has besides the obvious advantage of the reuse of spectral resources. Notwithstanding, the implementation of IBFD systems also represents a technological challenge [12]. That is why in this 
article, we intend to demonstrate the feasibility of a synchronization loop using IBFD on actual hardware SDR platforms.

Figure 1 shows a conceptual diagram of the target scenario in which two satellites perform synchronization and transmit (or receive) towards a common target.

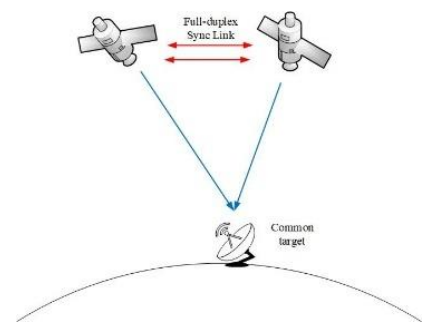

Figure 1. General system diagram for the implemented testbed

The SDR testbed implements a master/slave architecture using IBFD, where each of the nodes performs self-signal detection and cancelation, while the channel is emulated using an SDR satellite channel emulator developed in previous projects [13]. The experimental results show the accuracy of the signal cancellation procedure and demonstrate the feasibility of the IBFD synchronization between the two nodes, extendible to the application in scenarios with more than two nodes.

\section{SYSTEM FOR FULL-DUPLEX SYNCHRONIZATION LOOP}

The system for remote clock synchronization proposed in this work consists of:

1) Two satellites with dual full-duplex transceivers.

2) A $6 x 6$ channel emulator matrix.

3) Two on-ground transceivers coined "targets."

The synchronization system must use some protocol with known pilot fields and a coherent operation over time. DVB$\mathrm{S} 2(\mathrm{X})$ is an excellent example since it is also widely used in satellite communications. The known pilot fields are used for frequency, time, and phase estimation and later compensation to achieve clock synchronization.

The synchronization protocol is based on a master-slave architecture. First, the master transmits its clock via the modulated known pilots to the slave. Then, the slave synchronizes its phase with the master signal and retransmits it using its own pilots. Finally, the master receives the slave signal, measures the phase difference, estimates the channel phase, and pre-compensates the phase of the transmitted pilots. This protocol reuses the same band thanks to the fullduplex capabilities of the satellite transceivers.

The communications channel in between the satellite-satellite and satellite-target transceivers is described using a 6x6 complex matrix $\mathbf{H}_{6 x 6}$. The full system can be modeled with the following equation:

$$
\left(\begin{array}{l}
S A T 1_{R X 1} \\
S A T 1_{R X 2} \\
S A T 2_{R X 1} \\
S A T 2_{R X 2} \\
T A R 1_{R X} \\
T A R 2_{R X}
\end{array}\right)=\mathbf{H}_{6 \times 6}\left(\begin{array}{l}
S A T 1_{T X 1} \\
S A T 1_{T X 2} \\
S A T 2_{T X 1} \\
S A T 2_{T X 2} \\
T A R 1_{T X} \\
T A R 2_{T X}
\end{array}\right)+\mathbf{n},
$$

where $\mathbf{n}$ is Additive Gaussian Noise (AWGN) and $\mathbf{H}_{6 x 6}$ is the channel matrix modeling free path losses, propagation phase delay, and Doppler frequency shifts. Another effect emulated using the $\mathbf{H}_{6 x 6}$ matrix is the auto-interference of the fullduplex transceivers, assuming that there is no saturation in the RF components.

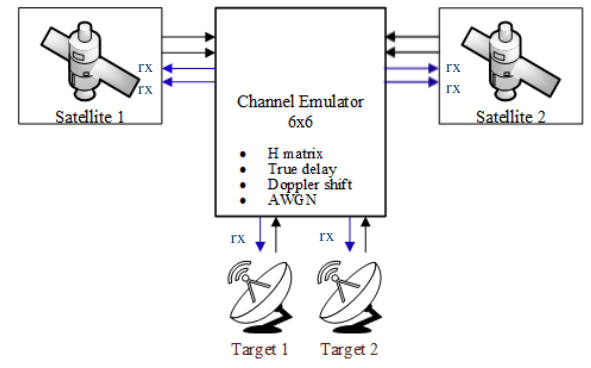

Figure 2. Block diagram of the SDR testbed implementation. The MIMO channel emulator implements the phase (H matrix), adjustable true delay, and adjustable Doppler effects.

At an initial stage, it is assumed that both targets are idle until the first satellite synchronization is done. Then, the satellites can operate coordinately to perform beamforming on one or both targets, while keeping tracking of the clock phase. Transmitters or receivers of targets may be enabled or disabled depending on the final application.

DUAL CARRIER POINT-TO-POINT SYNC

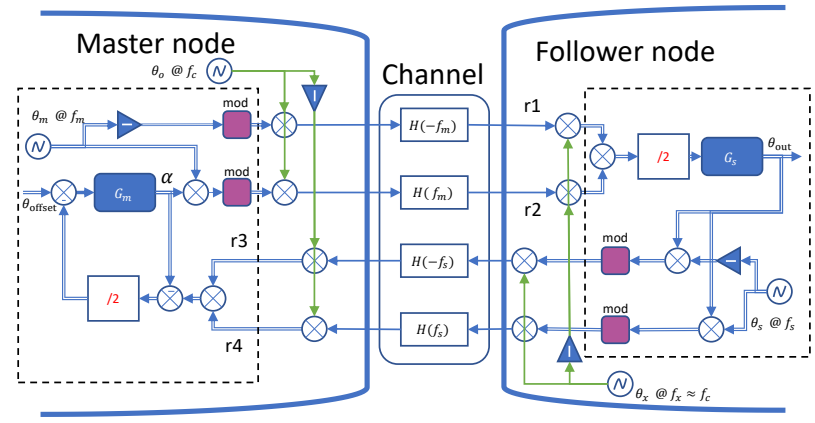

Figure 3. General dual-carrier synchronization scheme.

Figure 3. shows the synchronization scheme used between the two satellites, the master and the follower. 


\section{TESTBED DESCRIPTION}

The testbed consists of the commercially available SDR infrastructure developed by National Instruments (NI). The infrastructure consists, as shown in Figure 4, of a NI PXI (PCI EXtension for Instruments) 1085 chassis, used for the implementation of the MIMO satellite emulator. This channel emulator interconnects the two satellites and an additional receiver (User Terminal Emulator).

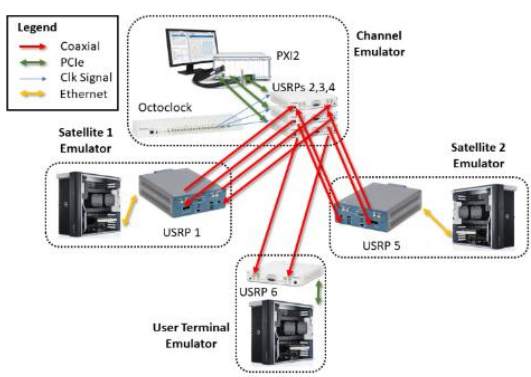

Figure 4 Laboratory testbed setup principal diagram.

The PXI centralizes the radio signals coming from up to 6 inputs taken using a set of NI USRP (Universal Software Radio Peripheral) 2954R, which is based on a Kintex FPGA from Xilinx. The satellite emulators are also implemented using USRPs, in particular, the standalone version 2974.

Each of the six outputs of the channel emulator is a linear combination of the available inputs, which is, in the present case, is 4 (but can also be 6, as seen in Figure 2). The synchronized operation of the two satellites enhances the performance of the received signals by the targets. Table 1 shows a description of the waveform parameters of the transmitted signal.

\begin{tabular}{|l|l|}
\hline PARAMETER & VALUE \\
\hline Standard & DVB-S2X, SuperFrame Format2 \\
\hline Modulations & QPSK (Up to 256APSK) \\
\hline Bandwidth & $3.2 \mathrm{Msymbols} / \mathrm{sec}$ (Up to 7.5 M) \\
\hline $\begin{array}{l}\text { Frequency separation } \\
\text { between carriers }\end{array}$ & $24 \mathrm{MHz}$ \\
\hline Pulse shaping & Root-Raised-Cosine \\
\hline Number of streams & 4 (2 per satellite) \\
\hline
\end{tabular}

Table 1 Transmitted signal parameters.

\section{RESULTS}

Figure 5 shows the spectrum with the four carriers seen by the channel emulator. It shows how the two carriers in one direction (master to follower) overlap with the two carriers coming in the opposite direction. This spectrum determines the maximum SNR available at the receivers, which is modified by a Gaussian noise artificially generated in the channel emulator.

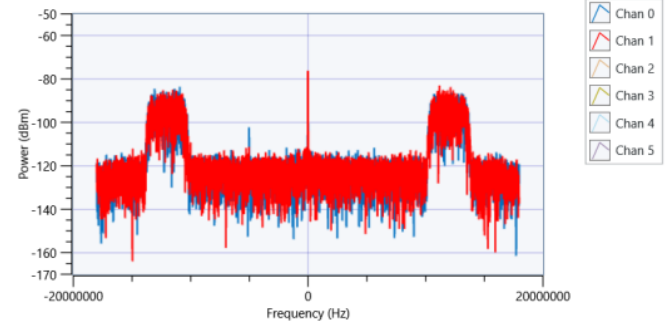

Figure 5. Spectrum measurement at the channel emulator.

We implemented the phase trackers in the receivers using $2^{\text {nd }}$ order digital PLLs in the FPGAs. After the round trip loop is closed, we can observe the phase variations applied to the transmitters in the master satellite, representing the phase over time of the full loop. Figure 6. shows the phase history for three different SNR values (set equal for the four receivers). For the three measurements, the PLL loop bandwidth is kept constant. The loop bandwidth should be later optimized as a function of the SNR. However, for the present experiment, the objective is not to optimize the loop performance but to demonstrate the concept.

Phase error measured at the Master

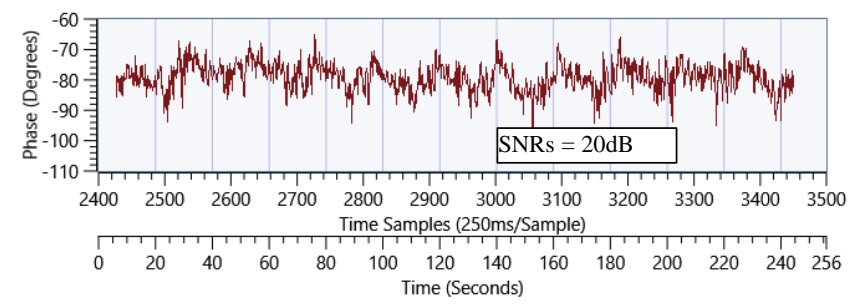

Phase error measured at the Master
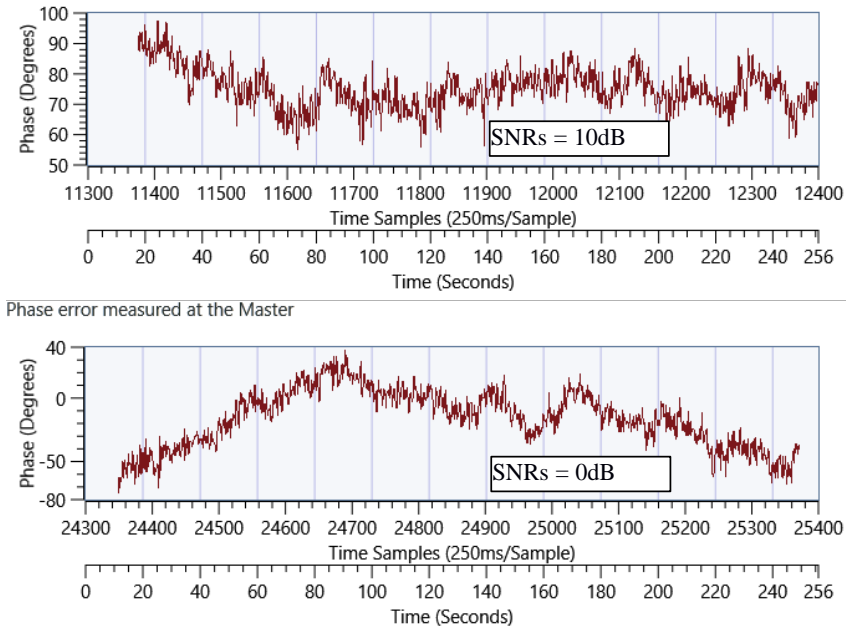

Figure 6. Phase error of the round trip loop compensated in the master for different SNR values.

In Figure 7 the phase difference between two carriers (the two overlapping carriers on the left of Figure 5) is observed in the 
TARGET receivers. Here it can be seen how the phase offset is stable even in the presence of the Doppler offset injected in the channel emulator $(0$ and $5 \mathrm{~Hz})$.

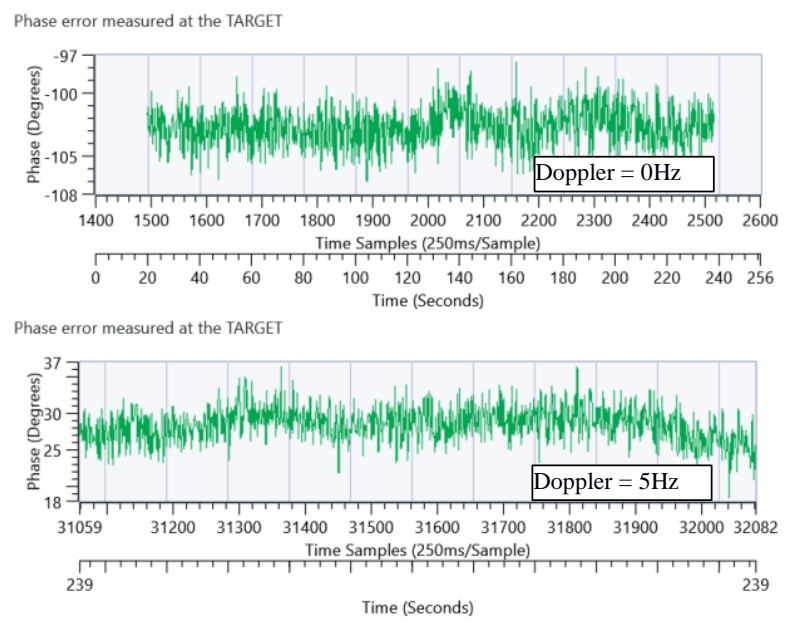

Figure 7. Phase difference between two overlapping carriers received by the target for two different Doppler offsets, $0 \mathrm{~Hz}$, and $5 \mathrm{~Hz}$. SNR=20dB in both tracking loop receivers and target receivers (for a total of 6 receivers).

\section{CONCLUSIONS}

The concept of digital synchronization scheme using dual DVB-S2X carriers was successfully validated in hardware. The next steps in the prototype development will include adaptive optimization of the system parameters depending on the scenario conditions.

\section{ACKNOWLEDGMENTS}

This work was supported by the Fond National de la Recherche Luxembourg, under the CORE project COHESAT: Cognitive Cohesive Networks of Distributed Units for Active and Passive Space Applications.

\section{REFERENCES}

[1] G. Krieger and A. Moreira, "Spaceborne bi- and multistatic SAR: Potential and challenges," IEE Proc. Radar, Sonar Navig., vol. 153, no. 3, pp. 184186, Jun. 2006.

[2] H. Fiedler et al., "The TanDEM-X mission: an overview," in 2008 International Conference on Radar, 2008, pp. 60-64.

[3] P. Lopez-Dekker, H. Rott, P. Prats-Iraola, B. Chapron, K. Scipal, and E. De Witte, "Harmony: an Earth Explorer 10 Mission Candidate to Observe Land, Ice, and Ocean Surface Dynamics," in IGARSS 2019 - 2019 IEEE International Geoscience and Remote Sensing Symposium, 2019, pp. 8381-8384.

[4] J. Matar, M. Rodriguez-Cassola, G. Krieger, P.
Lopez-Dekker, and A. Moreira, "MEO SAR: System Concepts and Analysis," IEEE Trans. Geosci. Remote Sens., pp. 1-12, 2019.

Z. Yu, J. Chen, C. Li, Z. Li, and Y. Zhang, "Concepts, properties, and imaging technologies for GEO SAR," in MIPPR 2009: Multispectral Image Acquisition and Processing, 2009, vol. 7494, p. 749407.

A. Monti Guarnieri, A. Broquetas, A. Recchia, F. Rocca, and J. Ruiz-Rodon, "Advanced Radar Geosynchronous Observation System: ARGOS," IEEE Geosci. Remote Sens. Lett., vol. 12, no. 7, pp. 1406-1410, Jul. 2015.

A. K. Sugihara El Maghraby, A. Grubisic, C. Colombo, and A. Tatnall, "A Novel Interferometric Microwave Radiometer Concept Using Satellite Formation Flight for Geostationary Atmospheric Sounding," IEEE Trans. Geosci. Remote Sens., vol. 56, no. 6, pp. 3487-3498, Jun. 2018.

A. K. S. El Maghraby, A. Grubisic, C. Colombo, and A. Tatnall, "A Hexagonal Pseudo-polar FFT for Formation-Flying Interferometric Radiometry," IEEE Geosci. Remote Sens. Lett., vol. 16, no. 3, pp. 432-436, Mar. 2019.

[9] W. J. Blackwell, "Technology Evolution to Enable High-Performance Cubesat Radiometry Missions," IGARSS 2019 - 2019 IEEE Int. Geosci. Remote Sens. Symp., pp. 5078-5081, Jul. 2019.

[10] L. Feng, Q. Li, and Y. Li, "Imaging with 3-D Aperture Synthesis Radiometers," IEEE Trans. Geosci. Remote Sens., vol. 57, no. 4, pp. 2395-2406, 2019.

[11] J. C. Merlano-Duncan, J. Querol, A. Camps, S. Chatzinotas, and B. Ottersten, "Architectures and Synchronization Techniques for Coherent Distributed Remote Sensing Systems," IGARSS 2019 - 2019 IEEE Int. Geosci. Remote Sens. Symp., pp. 8875-8878, Jul. 2019.

[12] M. Duarte, C. Dick, and A. Sabharwal, "Experimentdriven characterization of full-duplex wireless systems," IEEE Trans. Wirel. Commun., vol. 11, no. 12, pp. 4296-4307, 2012.

[13] J. Duncan et al., "Hardware Precoding Demonstration in Multi-Beam UHTS Communications under Realistic Payload Characteristics," in 37th International Communications Satellite Systems Conference (ICSSC 2019), 2019, pp. 2-4.

[14] S. Felici-Castell, E. Navarro, J. Pérez-Solano, J. Segura-García, and M. García-Pineda, "Practical Considerations in the Imp. of Collaborative Beamforming on Wireless Sensor Networks," Sensors, vol. 17, no. 2, p. 237, Jan. 2017. 